\title{
ON THEOREMS CONNECTING MELLIN AND HANKEL TRANSFORMS
}

\author{
VIRENDRA KUMAR
}

\begin{abstract}
In the present paper four theorems connecting Mellin and Hankel transforms are established. The theorems are general in nature. As application, four integrals involving special functions are obtained. It is obvious from the examples that we can evaluate integrals involving special functions with the help of the theorems established in this paper. Otherwise it is difficult to evaluate such type of integrals.
\end{abstract}

Mathematics subject classification (2010): 44A05. function.

Keywords and phrases: Mellin transform, Hankel transform, Laplace transform, Bessel function, Struve's

\section{REFERENCES}

[1] A. ErdéLyi, W. Magnus, F. Oberhettinger and F. G. Tricomi, Higher Transcendental Functions, Vol. I, McGraw-Hill, New York, Toronto and London 1953.

[2] A. Erdélyi, W. Magnus, F. Oberhettinger and F. G. Tricomi, Higher Transcendental Functions, Vol. II, McGraw-Hill, New York, Toronto and London 1953.

[3] A. Erdélyi, W. Magnus, F. Oberhettinger and F. G. Tricomi, Tables of Integral Transforms, Vol. I, McGraw-Hill, New York, Toronto and London 1954.

[4] A. ERdélyi, W. Magnus, F. Oberhettinger and F. G. TRicomi, Tables of Integral Transforms, Vol. II, McGraw-Hill, New York, Toronto and London 1954.

[5] L. LANDAU, Monotonicity and bounds for Bessel functions, Proceedings of the Symposium on Mathematical Physics and Quantum Field Theory (Berkeley, California: June 11-13, 1999)(Warchall. H, Editor), 147-154(2000); Electron. J. Differential Equations, Southwest Texas State University, San Marcos, Texas, 4 (2002).

[6] L. J. LandaU, Bessel functions: Monotonicity and bounds, Journal of the London Mathematical Society, 61, 1 (2000), 197-215.

[7] F. Oberhettinger, Tables of Mellin Transforms, Springer-Verlag, Berlin, Germany, 1974.

[8] A. P. Prudnikov, Yu. A. Brychiov And O. I. Marichev, Integrals and series. Volume 2. Elementary functions, Gordon and Breach science publishers, New York, 1986.

[9] A. P. Prudnikov, Yu. A. Brychkov And O. I. Marichev, Integrals and series. Volume 2. Special functions, Gordon and Breach science publishers, New York, 1986.

[10] I. N. Sneddon, Fourier Transforms, McGraw-Hill, New York, 1951. 